This article is licensed under the Creative Commons Attribution-NonCommercial 4.0 International License (CC BY-NC) (http://www.karger.com/Services/OpenAccessLicense). Usage and distribution for commercial purposes requires written permission.

\title{
Complete Response to Chemotherapy against the Recurrence of Neuroendocrine Carcinoma of the Breast in the Anterior Mediastinal Lymph Nodes of a Patient on Hemodialysis: A Case Report
}

\author{
Emi Chikuie $^{a}$ Senichiro Yanagawa ${ }^{a}$ Hidehiro Tanji ${ }^{a}$ Shinya Kodama ${ }^{a}$ \\ Yukio Takeshima ${ }^{b}$ Kazuo Sumimoto ${ }^{a, c}$ \\ aDepartment of Surgery, Yoshida General Hospital, Akitakata, Japan; ${ }^{b}$ Department of \\ Pathology, Graduate School of Biomedical and Health Sciences, Hiroshima University, \\ Hiroshima, Japan; 'Department of Gastroenterological and Transplant Surgery, Graduate \\ School of Biomedical and Health Sciences, Hiroshima University, Hiroshima, Japan
}

\section{Keywords}

Hemodialysis · Neuroendocrine carcinoma of breast · Chemotherapy

\begin{abstract}
In general, the cancer-bearing rate of patients on dialysis is considered to be higher than that of healthy individuals. There is no established chemotherapy regimen for patients on hemodialysis (HD), but some reports have described chemotherapy administration in such patients according to HD conditions, residual renal function, and the drug metabolic pathway. We present a case involving a 76-year-old female patient on HD who underwent chemotherapy for mediastinal lymph node recurrence of neuroendocrine carcinoma of the breast (NECB) using
\end{abstract}


5-fluorouracil, epirubicin, and cyclophosphamide (FEC 100) and docetaxel (DTX). The patient showed a complete response to the treatment and survived longer than expected. This is the first report demonstrating a complete response to chemotherapy for the recurrence of primary NECB with the patient having survived for a long time after treatment.

(C) 2019 The Author(s)

Published by S. Karger AG, Basel

\section{Introduction}

Prolonged life expectancy and evolution of medical technology have increased the detection and treatment response rates for patients with malignant tumors [1]. The incidence of malignancy in patients on hemodialysis (HD) is higher than that in healthy individuals; thus, the need for treatment in patients on HD with a malignancy is increasing [2]. Primary neuroendocrine carcinoma of the breast (NECB) is rare, with an incidence of under $0.1 \%$ from among all breast carcinomas. Only a limited number of studies on NECB have been reported in the literature [3].

In this study, we report a case involving a patient on HD with mediastinal lymph node recurrence of NECB who was treated using chemotherapy. Remission was noted, and no recurrence has been observed for forty-two months.

\section{Case Report}

A 76-year-old woman had a history of left NECB, which was classified as pT2N1M0 stage IIB according to the Union for International Cancer Control (UICC) tumor node metastasis (TNM) classification. The patient was admitted to our hospital with complaints of a mass in the right breast without axial lymph node swelling on computed tomography (CT) (Fig. 1a). No associated pain, nipplagnete discharge, skin change, or palpable lymphadenopathy was noted. Contrast-enhanced mic resonance imaging (MRI) showed a mass with early arterial enhancement and gradual washout measuring $9.3 \times 5.8 \times 10.7 \mathrm{~mm}$ (Fig. 1b). Right breast mastectomy and axillary lymph node dissection were performed. The final pathologic diagnosis was NECB with small polygonal cells having a small amount of cytoplasm, high nuclear-cytoplasmic ratio, and finely granular chromatin (Fig. 2). According to the UICC TNM classification, it was classified as pT1N0M0 stage IA. A diagnosis of non-intramammary metastasis was made based on the results of immunostaining: chromogranin A (-), synaptophysin $(+)$, Leu7 $(-)$, CD56 (-) in the left NECB, and chromogranin A (-), synaptophysin (-), Leu7 (+), CD56 (-) in the right NECB. Six months after the operation, the patient initially underwent HD owing to unexplained chronic renal failure and continued the treatment as an outpatient. Forty months after a right breast mastectomy, CT revealed anterior mediastinal lymph node recurrence (Fig. $3 a)$. Hence, we considered the recurrence of NECB and decided to initiate chemotherapy to prevent further recurrence. Based on the past literature on the chemotherapy dosage for patients on HD, a chemotherapy regimen with six cycles of FEC 100 - consisting of fluorouracil (5-FU, $500 \mathrm{mg} / \mathrm{m}^{2}$ ), epirubicin (EPI, $70 \mathrm{mg} / \mathrm{m}^{2}$ ), and cyclophosphamide (CPA, $350 \mathrm{mg} / \mathrm{m}^{2}$ ) and three cycles of docetaxel (DTX, $60 \mathrm{mg} / \mathrm{m}^{2}$ ) was initiated (Table 1). After the third course 


\section{Case Reports in Oncology}

Case Rep Oncol 2019;12:205-210

DOI: $10.1159 / 000497479$

(c)

2019 The Author(s). Published by S. Karger AG, Base www.karger.com/cro

Chikuie et al.: Complete Response to Chemotherapy against the Recurrence of

Neuroendocrine Carcinoma of the Breast

of DTX treatment, grade 3 neutropenia was observed; hence, the chemotherapy was discontinued. CT was performed after the chemotherapy and anterior mediastinal lymph node recurrence was not detected (Fig. 3b). The patient responded well to the chemotherapy. Fortytwo months after the chemotherapy, no tumor recurrence was detected on CT.

\section{Discussion}

The population of individuals undergoing dialysis is over 0.3 million in Japan [1]. Owing to the prolonged prognosis of patients on HD, the need for treatment for malignancy in such patients is increasing. Malignancy is one of the major causes of death among patients on HD $[4,5]$. However, there are no guidelines for the administration of chemotherapy for malignancy in patients on HD. Currently, the dosing regimens are individually administered according to information from previous case reports or the available literature, and dialysis conditions [6]. Patients with end-stage renal disease display enhanced genomic damage that may have pathophysiological relevance for cancer development. These patients may need drug dosage reduction to avoid overdosage and drug toxicity, and drug clearance by dialysis must be taken into account for appropriate chemotherapy timing.

In this case, there was no biopsy for mediastinal lymph node recurrence. Therefore, it was not possible to confirm the recurrence of NECB, but it was considered as such from the clinical course. A regimen for NECB has not been established; however, there are some reports that chemotherapy for breast cancer (BC) can also be used for patients with NECB; therefore, we used the regimen of FEC 100 and DTX [7-9].

We referred to the report of Janus et al. and introduced the FEC 100 regimen (5-FU, EPI, and CPA) [6]. The standard dose of 5-FU was used while EPI and CPA doses were reduced to $70 \%$ of the standard dose. During the 3-week course, three drugs were administered after the session on the first HD day. During the six cycles of FEC 100 chemotherapy, the side effects of grade 3 neutropenia or higher were not observed, as noted based on the Common Terminology Criteria for Adverse Events (CTCAE) v4.0. After completion of the FEC 100 chemotherapy, the DTX dose was reduced to $80 \%$ of the standard dose during the 3-week course. After three courses, DTX administration was stopped because of the incidence of grade 3 neutropenia. During this time, mediastinal lymph node metastasis was not observed in the CT.

There is no established standard treatment protocol because primary NECB is rarely reported. The optimal management strategy for the disease remains unknown; however, the treatment is similar to that for other conventional types of invasive BC [10]. The patient underwent FEC 100 and DTX chemotherapy for distinct metastasis, and it is often used to treat patients with BC. The standard chemotherapy for advanced BC was effective in our patient.

In this case, the patient has remained free from the disease as of the last follow-up at thirty-six months after the chemotherapy. To our knowledge, this is the first report demonstrating the improvement in the survival and $\mathrm{BC}$ recurrence in a patient on HD undergoing chemotherapy. We believe that this case report is useful in providing information on treatment options for patients on HD.

Even in patients on HD, such a recurrence can be cured by chemotherapy due to a careful individual response. 


\section{Statement of Ethics}

Written informed consent was obtained from the patient.

\section{Disclosure Statement}

The authors have no conflicts of interest to declare.

\section{Author Contributions}

EC and SY made substantial contributions to the concept and design of the study, and data acquisition and interpretation. HT, SK, YT, and KS were involved in manuscript drafting and critical revision for important intellectual content. SY gave the final approval for the version to be published. All authors read and approved the final manuscript.

\section{References}

1 Liyanage T, Ninomiya T, Jha V, Neal B, Patrice HM, Okpechi I, et al. Worldwide access to treatment for endstage kidney disease: a systematic review. Lancet. 2015 May;385(9981):1975-82.

2 Maisonneuve P, Agodoa L, Gellert R, Stewart JH, Buccianti G, Lowenfels AB, et al. Cancer in patients on dialysis for end-stage renal disease: an international collaborative study. Lancet. 1999 Jul;354(9173):93-9.

3 Wang J, Wei B, Albarracin CT, Hu J, Abraham SC, Wu Y. Invasive neuroendocrine carcinoma of the breast: a population-based study from the surveillance, epidemiology and end results (SEER) database. BMC Cancer. 2014 Mar;14(1):147.

4 Masakane I, Nakai S, Ogata S, Kimata N, Hanafusa N, Hamano T, et al. An overview of regular dialysis treatment in Japan (as of 31 December 2013). Ther Apher Dial. 2015 Dec;19(6):540-74.

5 de Jager DJ, Grootendorst DC, Jager KJ, van Dijk PC, Tomas LM, Ansell D, et al. Cardiovascular and noncardiovascular mortality among patients starting dialysis. JAMA. 2009 0ct;302(16):1782-9.

6 Janus N, Thariat J, Boulanger H, Deray G, Launay-Vacher V. Proposal for dosage adjustment and timing of chemotherapy in hemodialyzed patients. Ann Oncol. 2010 Jul;21(7):1395-403.

7 López-Bonet E, Alonso-Ruano M, Barraza G, Vazquez-Martin A, Bernadó L, Menendez JA. Solid neuroendocrine breast carcinomas: incidence, clinico-pathological features and immunohistochemical profiling. Oncol Rep. 2008 Dec;20(6):1369-74.

8 Nozoe T, Sueishi K, Mori E, Iguchi T, Egashira A, Adachi E, et al. Primary neuroendocrine carcinoma of the breast: report of a case. Surg Today. 2011 Jun;41(6):829-31.

9 Zhang JY, Chen WJ. Bilateral primary breast neuroendocrine carcinoma in a young woman: report of a case. Surg Today. 2011 Nov;41(11):1575-8.

10 Adams RW, Dyson P, Barthelmes L. Neuroendocrine breast tumours: breast cancer or neuroendocrine cancer presenting in the breast? Breast. 2014 Apr;23(2):120-7. 


\section{Case Reports in Oncology}

Case Rep Oncol 2019;12:205-210

DOI: $10.1159 / 000497479$

(C) 2019 The Author(s). Published by S. Karger AG, Basel www.karger.com/cro

Chikuie et al.: Complete Response to Chemotherapy against the Recurrence of

Neuroendocrine Carcinoma of the Breast
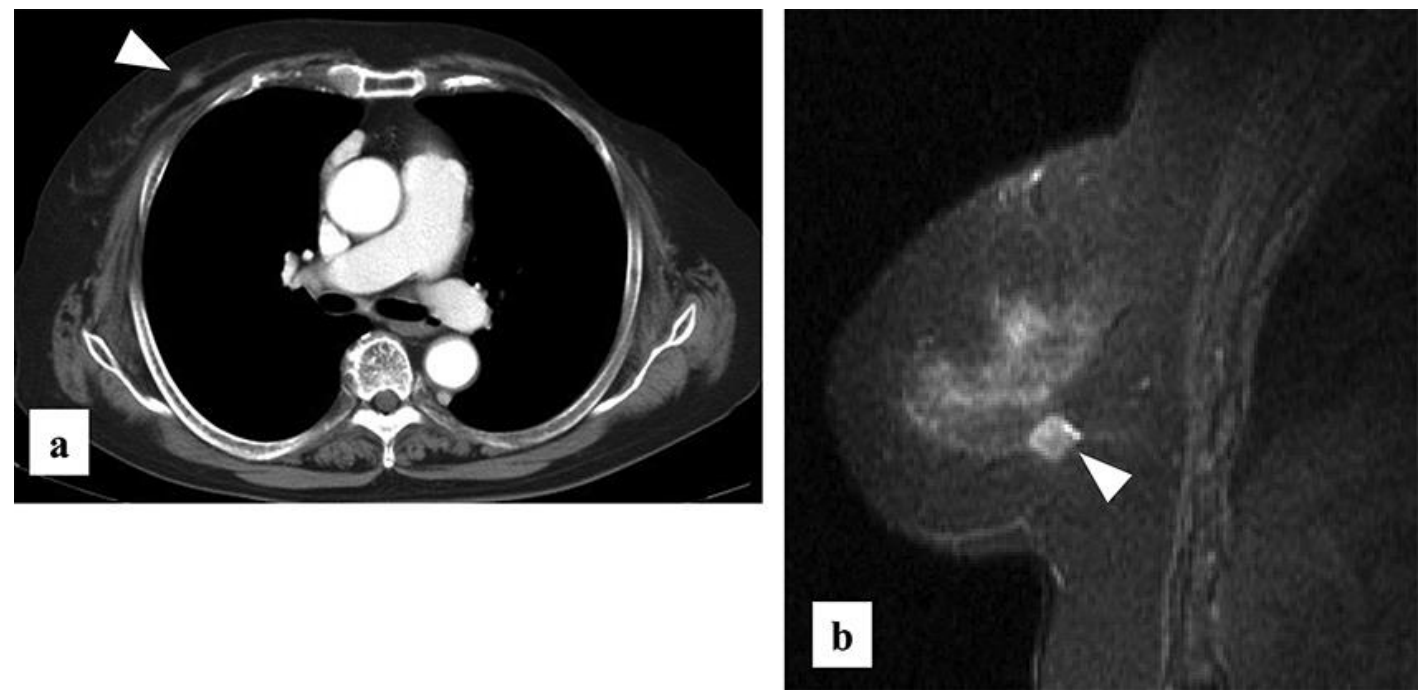

Fig. 1. (a) CT scan showing a high contrast mass, but no axial lymph node swelling. Abnormal findings were observed in distant organs. (b) Contrast-enhanced MRI showing a mass in the same region that had early arterial enhancement and gradual washout.

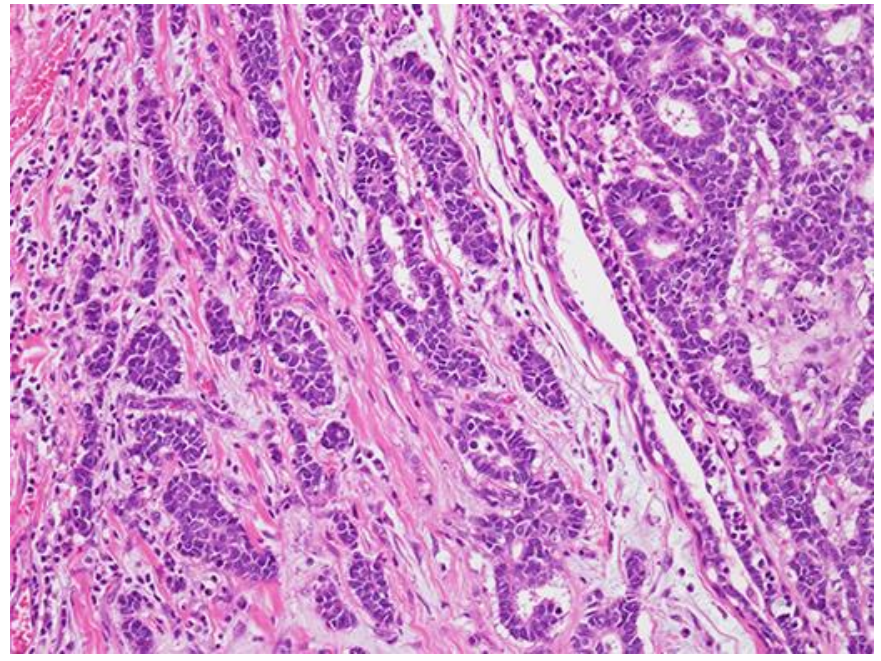

Fig. 2. The tumor shows trabecular growth patterns with a rosette formation. 


\section{Case Reports in Oncology}
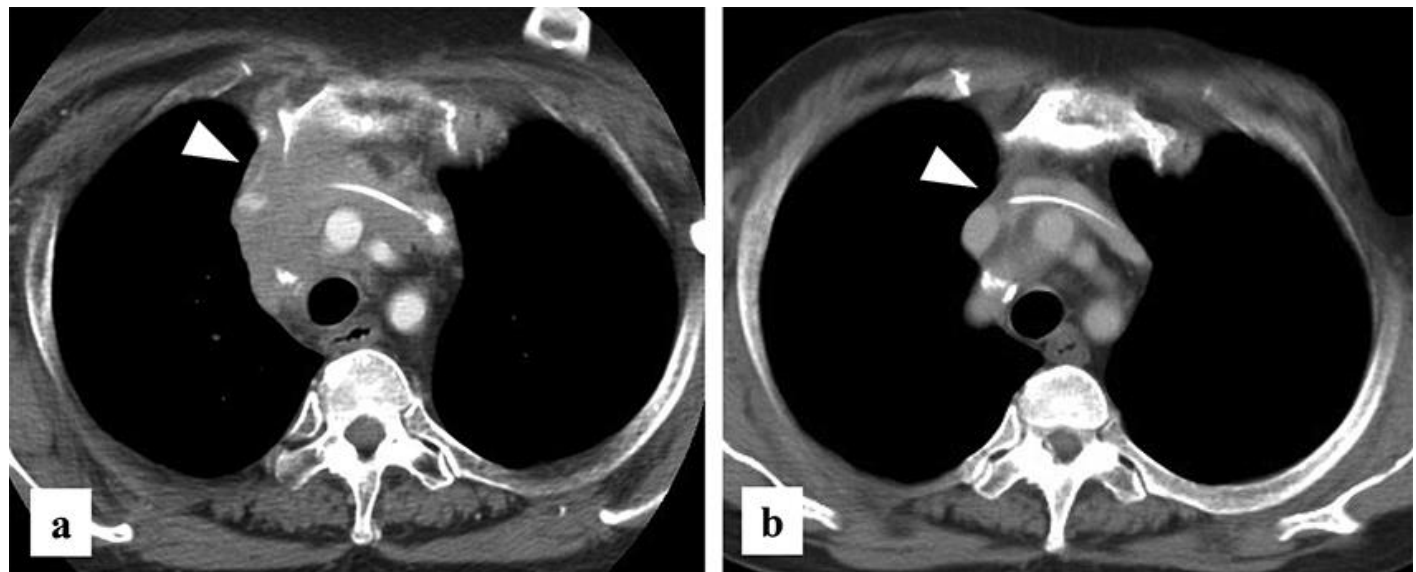

Fig. 3. (a) CT reveals the presence of anterior mediastinal lymph node metastasis. (b) After chemotherapy, CT reveals no lymph node metastases.

Table 1. Dosing schedule of FEC 100 and DTX (3-week course)

\begin{tabular}{|c|c|c|c|c|c|c|c|c|c|c|}
\hline Day & 1 & 3 & 5 & 8 & 10 & 12 & 15 & 17 & 19 & 21 \\
\hline HD & $\downarrow$ & $\downarrow$ & $\downarrow$ & $\downarrow$ & $\downarrow$ & $\downarrow$ & $\downarrow$ & $\downarrow$ & $\downarrow$ & $\downarrow$ \\
\hline EPI & $\downarrow$ & & & & & & & & & \\
\hline CPA & $\downarrow$ & & & & & & & & & \\
\hline 5-FU & $\downarrow$ & & & & & & & & & \\
\hline Day & 1 & 3 & 5 & 8 & 10 & 12 & 15 & 17 & 19 & 21 \\
\hline HD & $\downarrow$ & $\downarrow$ & $\downarrow$ & $\downarrow$ & $\downarrow$ & $\downarrow$ & $\downarrow$ & $\downarrow$ & $\downarrow$ & $\downarrow$ \\
\hline
\end{tabular}

